\title{
Global Exponential Synchronization of Nonlinearly Coupled Complex Dynamical Networks with Time-Varying Coupling Delays
}

\author{
Yi-Ping Luo, ${ }^{1,2}$ Li Shu, ${ }^{1,2}$ and Bi-Feng Zhou ${ }^{3}$ \\ ${ }^{1}$ Hunan Provincial Key Laboratory of Wind Generator and Its Control, Hunan Institute of Engineering, Xiangtan, \\ Hunan 411104, China \\ ${ }^{2}$ The Cooperative Innovation Center of Wind Power Equipment and Energy Conversion, Xiangtan, Hunan 411104, China \\ ${ }^{3}$ Hunan Electrical College of Technology, Xiangtan, Hunan 411101, China \\ Correspondence should be addressed to Li Shu; shuli@hnie.edu.cn
}

Received 21 April 2017; Revised 28 June 2017; Accepted 2 July 2017; Published 9 August 2017

Academic Editor: David Arroyo

Copyright (c) 2017 Yi-Ping Luo et al. This is an open access article distributed under the Creative Commons Attribution License, which permits unrestricted use, distribution, and reproduction in any medium, provided the original work is properly cited.

\begin{abstract}
This paper focuses on the global exponential synchronization problem of nonlinearly coupled complex dynamical networks with time-varying coupling delays. Several simple and generic global exponential synchronization criteria are derived based on the Lyapunov stability theory and the Dini derivatives using the Halanay and generalized Halanay inequalities. These criteria rely on system parameters alone and can be used conveniently in practical applications. In addition, the system parameters do not satisfy the conditions of the proposed criteria. That is, the system itself cannot synchronize. However, system synchronization can be achieved by adding the appropriate feedback controllers, thereby providing a practical and effective control method for complex dynamical networks. An estimation method of exponential convergence rate is also presented. Finally, the effectiveness of the proposed criteria is verified through numerical simulations.
\end{abstract}

\section{Introduction}

Complex dynamical networks have attracted considerable attention in the past several decades because of their potential applications in diverse fields, such as science, engineering, and societal systems [1-4]. In fact, a variety of real-world systems, such as the Internet, food webs, and ecological, neural, and social networks, can be described by complex dynamical networks. Consequently, synchronization, which is a typical collective behavior of complex dynamical networks, has become active popular research topic. Synchronization in dynamical networks has been extensively investigated by researchers from various fields [5-13].

Research on the synchronization of complex networks has two main aspects. In some cases, complex dynamical networks can achieve synchronization through their topological structure, communication quality, and the interaction of the intrinsic dynamical behavior of their nodes [1417]. Wang and Chen $[14,15]$ were the first to propose a method for measuring synchronization capability by calculating eigenvalues; that is, synchronization will be achieved if the coupling strength is adequate. A previous study [16] utilized a novel Lyapunov-Krasovskii function and the Kronecker product and discussed synchronization problems for an array of coupled complex discrete-time networks. Huang et al. [17] introduced the complex dynamical network model with partial information transmission, for which certain synchronization criteria were derived by using an efficient decomposition method. However, the synchronization criteria in most existing works contain unknown parameters, thereby making system synchronization difficult for workers to verify.

A control action is introduced in the dynamical node to drive a complex dynamical network into synchronization if the network cannot synchronize by itself. Researchers have proposed several effective control methods, and significant conclusions have been obtained [18-25]. Guaranteed cost synchronization for complex networks was addressed 
in [18] by designing a dynamic feedback controller with guaranteed cost synchronization. Zhou et al. [19] investigated the local and global adaptive synchronizations of uncertain complex dynamical networks. Their hypotheses and adaptive controller design for network synchronization are rather simple in form. The global exponential synchronization of complex-valued dynamical networks with multiple timevarying delays and stochastic perturbations were considered in [20] in the design of a time-delayed impulsive control scheme. Cheng et al. [21] found that complex networks with the Watts-Strogatz or scale-free BA random topological architecture can be synchronized more easily than regular systems by pin-controlling fewer nodes. Intermittent pinning controllers were applied in [22] to synchronize interacting clusters of linearly coupled heterogeneous linear systems and nonlinear oscillators under a general coupling topology. These studies use the coupling matrix between nodes to affect synchronization, thereby creating the advantage of fully utilizing the information between nodes. Deficiency increases the number of adjustment parameters that affect system synchronization. Thus, verifying system synchronization can be difficult. However, system synchronization can be verified easily using a method based on these findings. This method relies only on the parameters of the system itself and does not need any other adjustment parameter, thereby reducing delays and providing convenience for controller design. However, few results on this study have been reported.

The key to studying complex networks is building a network model that can exactly describe a real network system. Delays should be considered in establishing a network model because of the finite speed of information transmission and traffic congestion [26, 27]. Time delay commonly changes over time during dynamic change. Fixed delay is an ideal state of time-varying delay. Therefore, time-varying delay should be considered when studying complex networks [28-32].

Inspired by the above discussion, this paper aims to investigate the global exponential synchronization of a class of complex dynamical networks with time-varying delay. We consider a complex network model composed of nonlinear coupling nodes and has unknown but bounded nonlinear vector functions and time-varying delayed and nondelayed couplings. Several simple global exponential synchronization criteria are derived from the Lyapunov stability theory and the Dini derivatives using the Halanay and generalized Halanay inequalities. These criteria rely on system parameters alone and can be used conveniently in practical applications. In addition, the system parameters do not satisfy the conditions of the proposed criteria, that is, the system itself cannot achieve synchronization. However, the system can be driven to synchronize by adding appropriate feedback controllers. These conditions provide a practical and effective control method for complex network systems. Finally, the validity of the proposed scheme is verified by numerical simulations.

The rest of this paper is organized as follows. Section 2 introduces the complex dynamical network model and provides certain preliminaries. Section 3 presents the criteria for ensuring the global exponential synchronization of complex dynamical networks with time-varying delay and an estimation method for exponential convergence rate. Section 4 presents the numerical simulations used to validate the proposed scheme. Finally, Section 5 concludes this paper.

\section{Problem Formulation and Preliminaries}

A complex network with $N$ identical time-varying delayed dynamical nodes with nonlinear couplings is considered. Each node of the network has $n$ dimensions. The state of the $i$ th node can be described as follows:

$$
\begin{aligned}
\dot{x}_{i}(t)= & f\left(x_{i}(t)\right)+c_{1} \sum_{j=1}^{N} c_{i j} g_{j}\left(x_{j}(t)\right) \\
& +c_{2} \sum_{j=1}^{N} d_{i j} g_{j}\left(x_{j}(t-\tau(t))\right), \quad i=1,2, \ldots, N,
\end{aligned}
$$

where $x_{i}(t)=\left(x_{i 1}(t), x_{i 2}(t), \ldots, x_{i n}(t)\right)^{T} \in R^{n}$ is the state vector of the $i$ th node, $f(x) \in R^{n}$ is a continuously nonlinear vector-valued function that describes the dynamics of a node, and $g(x) \in R^{n}$ is a continuous nonlinear coupling function. The coupling time delay $\tau(t)$ is unknown but is bounded by a known constant, that is, $0 \leq \tau(t) \leq \tau$, where positive constants $c_{1}$ and $c_{2}$ are the coupling strengths. $C=\left(c_{i j}\right)_{N \times N}$ and $D=\left(d_{i j}\right)_{N \times N}$ are the coupling configuration matrices. If node $i$ and $j(j \neq i)$ are connected, then $c_{i j}>0, d_{i j}>0$; otherwise, $c_{i j}=0, d_{i j}=0$. The diagonal elements of matrices $C$ and $D$ are defined as $c_{i i}=-\sum_{j=1, j \neq i}^{N} c_{i j}, d_{i i}=-\sum_{j=1, j \neq i}^{N} d_{i j}$.

Definition 1. Generally, when $t \rightarrow \infty, x_{1}(t), x_{2}(t), \ldots$, $x_{N}(t) \rightarrow s(t) . s(t) \in R^{n}$ is the solution of the following isolated node system (2). The complex dynamic system (1) synchronizes with the homogenous trajectory

$$
\dot{s}(t)=f(s(t)) .
$$

Let $e_{i}(t)=x_{i}(t)-s(t)$ be the synchronization error. We obtain the error system on the basis of network (1) and (2) as follows:

$$
\begin{aligned}
\dot{e}_{i}(t)= & \dot{x}_{i}(t)-\dot{s}(t) \\
= & f_{i}\left(x_{i}(t)\right)-f(s(t))+c_{1} \sum_{j=1}^{N} c_{i j} \phi_{j}\left(e_{j}(t)\right) \\
& +c_{2} \sum_{j=1}^{N} d_{i j} \phi_{j}\left(e_{j}(t-\tau(t))\right),
\end{aligned}
$$

where $\phi_{j}\left(e_{j}(t)\right)=g_{j}\left(x_{j}(t)\right)-g(s(t)), \phi_{j}\left(e_{j}(t-\tau(t))\right)=$ $g_{j}\left(x_{j}(t-\tau(t))\right)-g(s(t-\tau(t)))$.

Definition 2. $\left|x_{i}(t)\right|=\left(\left|x_{i 1}(t)\right|,\left|x_{i 2}(t)\right|, \ldots,\left|x_{i n}(t)\right|\right)^{T} \in R^{n}$, $i=1,2, \ldots, N$, where $|x|$ denotes the absolute value of $x$.

Definition 3. A positive constant $\gamma$ and vector $M=\left(m_{1}, m_{2}\right.$, $\left.\ldots, m_{n}\right)^{T}>0$ exist if the vector function $e_{i}(t)=\left(e_{i 1}(t), e_{i 2}(t)\right.$, $\left.\ldots, e_{i n}(t)\right)^{T} \in R^{n}$ satisfies

$$
\left|e_{i}(t)\right| \leq M \exp (-\gamma t)
$$


The error system (3) is globally and exponentially stable, which implies that the complex dynamical network (1) achieves global exponential synchronization.

Remark 4. If the state vector of the error system (3) satisfies the conditions of Definition 3, then $\left\|e_{i}(t)\right\|=\sqrt{e_{i}^{T}(t) e_{i}(t)}$, where $\|\cdot\|$ indicates the Euclidean norm, is globally and exponentially stable.

Definition 5 (see [33]). The Dini derivatives of $f$ at $t_{0} \in(\alpha, \beta)$ for the vector-valued function $f:(\alpha, \beta) \rightarrow R, t \rightarrow f(t)$ are defined as

$$
D^{+} f\left(t_{0}\right)=\lim _{t \rightarrow t_{0}+} \sup \frac{f(t)-f\left(t_{0}\right)}{t-t_{0}}
$$

Definition 6 (see [33]). A matrix $A=\left(a_{i j}\right)_{N \times N}$ is called an $M$ matrix if it satisfies $a_{i i}>0, a_{i j} \leq 0, i \neq j, A^{-1} \geq 0$. According to the $M$-matrix property, a constant $\theta_{j}>0$ exists such that $\sum_{j=1}^{N} \theta_{j} a_{i j}>0, i=1,2, \ldots, N$.

Assumption 7. The nonlinear function $f\left(x_{i}\right), g\left(x_{i}\right)$ is assumed to satisfy the uniform semi-Lipschitzian condition, where constants $\sigma_{i}$ and $l_{i}$ satisfy

$$
\begin{aligned}
& 0 \leq \frac{f\left(\xi_{1}\right)-f\left(\xi_{2}\right)}{\xi_{1}-\xi_{2}} \leq \sigma_{i}, \quad i=1,2, \ldots, N, \\
& 0 \leq \frac{g\left(\xi_{1}\right)-g\left(\xi_{2}\right)}{\xi_{1}-\xi_{2}} \leq l_{i}, \quad i=1,2, \ldots, N .
\end{aligned}
$$

\section{Main Results}

3.1. Global Exponential Synchronization of System (1) Using the Halanay Inequality

Lemma 8 (Halanay [34]). Let $w(t):\left[t_{0}-\tau, \infty\right) \rightarrow[0, \infty)$ be a continuous function, where constants $a>b>0$ exist such that

$$
\dot{w}(t) \leq-a w(t)+b \bar{w}(t)
$$

holds for $t \geq t_{0}$, in which $\bar{w}(t)=\sup _{t-\tau \leq s \leq t} w(s), \tau \geq 0$, and then

$$
w(t) \leq \bar{w}\left(t_{0}\right) \exp \left\{-\gamma\left(t-t_{0}\right)\right\}, \quad t \geq t_{0},
$$

where $\gamma>0$ is the unique positive solution of the following equation:

$$
\gamma=a-b \exp \{\gamma \tau\} .
$$

Theorem 9. Supposing that Assumption 7 holds and satisfies the condition

$$
\min _{1 \leq i \leq N}\left(\varepsilon c_{1} l_{i}\right)>\max _{1 \leq i \leq N}\left(\sigma_{i}+\sum_{j=1, j \neq i}^{N} c_{1} l_{i} c_{i j}\right)+2 \max _{1 \leq i \leq N} c_{2} \lambda l_{i},
$$

where $\lambda$ is the maximum eigenvalue of the matrix $D=$ $\left(d_{i j}\right)_{N \times N}$, then the complex dynamical network (1) can achieve global exponential synchronization. $\mu / 2$ is the convergence rate, where $\mu$ is the unique positive solution of the equation, $\mu=a-b \exp (\mu t), a=2\left(\min _{1 \leq i \leq N}\left(\varepsilon c_{1} l_{i}\right)-\max _{1 \leq i \leq N}\left(\sigma_{i}+\right.\right.$ $\left.\left.\sum_{j=1, j \neq i}^{N} c_{1} l_{i} c_{i j}+c_{2} \lambda l_{i} / 2\right)\right), b=\max _{1 \leq i \leq N} c_{2} \lambda l_{i}$.

Proof. The Lyapunov function is constructed as follows:

$$
V(t)=\frac{1}{2} \sum_{i=1}^{N} e_{i}^{T}(t) e_{i}(t)
$$

Taking the derivative of $V(t)$ with respect to time $t$ along the solutions of error system (3) yields

$$
\begin{aligned}
\dot{V}(t)= & \sum_{i=1}^{N} e_{i}^{T}(t)\left(f\left(x_{i}(t)\right)-f(s(t))\right) \\
& +c_{1} \sum_{i=1}^{N} \sum_{j=1, j \neq i}^{N} c_{i j} e_{i}^{T}(t) \phi_{i}\left(e_{i}(t)\right) \\
& +c_{1} \sum_{i=1}^{N} c_{i i} e_{i}^{T}(t) \phi_{i}\left(e_{i}(t)\right) \\
& +c_{2} \sum_{i=1}^{N} \sum_{j=1}^{N} d_{i j} e_{i}^{T}(t) \phi_{j}\left(e_{j}(t-\tau(t))\right) .
\end{aligned}
$$

Assuming $c_{11}=c_{22}=\cdots=c_{N N}=-\varepsilon<0$, the assumption on the coupling matrix $C$ is not conservative. This because if $K=\left(k_{i j}\right)_{N \times N}$ is any square matrix that satisfies the conditions of the defined coupling configuration matrices, that is, $k_{i j} \geq 0$, $i \neq j, k_{i i}=-\sum_{j=1, j \neq i}^{N} k_{i j}$ and $k_{i i} \neq 0(i=1,2, \ldots, N)$, then all diagonal entries of the matrix $\widetilde{K}=\left(\widetilde{k}_{i j}\right)_{N \times N}=\left(k_{i j}\right)$ $\left.\sum_{j=1, j \neq i}^{N} k_{i j}\right)_{N \times N}$ are easily verified as -1 , and thus we can design the coupling matrix as $C=\varepsilon \widetilde{K}$.

Using Assumption $7\left(2 x^{T} y \leq x^{T} x+y^{T} y, \forall x, y \in R^{n}\right)$, the following inequalities can be estimated:

$$
\begin{aligned}
\dot{V} & (t) \leq \sum_{i=1}^{N} \sigma_{i} e_{i}^{T}(t) e_{i}(t)+c_{1} \sum_{i=1}^{N} \sum_{j=1, j \neq i}^{N} l_{i} c_{i j} e_{i}^{T}(t) e_{j}(t) \\
& -\varepsilon c_{1} \sum_{i=1}^{N} l_{i} e_{i}^{T}(t) e_{i}(t) \\
& +c_{2} \sum_{i=1}^{N} \sum_{j=1}^{N} l_{i} d_{i j} e_{i}^{T}(t) e_{j}(t-\tau(t)) \leq-\left(\min _{1 \leq i \leq N}\left(\varepsilon c_{1} l_{i}\right)\right. \\
& \left.-\max _{1 \leq i \leq N}\left(\sigma_{i}+\sum_{j=1, j \neq i}^{N} c_{1} l_{i} c_{i j}\right)\right) \sum_{j=1}^{N} e_{j}^{T}(t) e_{j}(t) \\
& +\max _{1 \leq i \leq N} \frac{c_{2} \lambda l_{i}}{2} \sum_{j=1}^{N} e_{j}^{T}(t) e_{j}(t)+\max _{1 \leq i \leq N} \frac{c_{2} \lambda l_{i}}{2}
\end{aligned}
$$




$$
\begin{aligned}
& \cdot \sum_{j=1}^{N} e_{j}^{T}(t-\tau(t)) e_{j}(t-\tau(t)) \leq-2\left(\min _{1 \leq i \leq N}\left(\varepsilon c_{1} l_{i}\right)\right. \\
& \left.-\max _{1 \leq i \leq N}\left(\sigma_{i}+\sum_{j=1, j \neq i}^{N} c_{1} l_{i} c_{i j}\right)+\max _{1 \leq i \leq N} \frac{c_{2} \lambda l_{i}}{2}\right) V(t) \\
& +\max _{1 \leq i \leq N} c_{2} \lambda l_{i} \bar{V}(t)=-a V(t)+b \bar{V}(t),
\end{aligned}
$$

where $\bar{V}(t)=(1 / 2) \sum_{i=1}^{N} \bar{e}_{i}^{T}(t) \bar{e}_{i}(t), \bar{e}_{i}(t)=\sup _{t-\tau \leq s \leq t} e_{i}(s)$.

According to the conditions of Theorem 9 and Lemma 8, we obtain

$$
V(t) \leq \bar{V}\left(t_{0}\right) \exp \left\{-\mu\left(t-t_{0}\right)\right\}
$$

where $\mu$ is the unique positive solution of the equation $\mu=$ $a-b \exp (\mu t)$

Thus, we obtain

$$
\begin{aligned}
& \sqrt{\sum_{i=1}^{N} e_{i}^{T}(t) e_{i}(t)} \\
& \quad \leq \sqrt{\sum_{i=1}^{N} \bar{e}_{i}^{T}\left(t_{0}\right) \bar{e}_{i}\left(t_{0}\right) \exp \left\{-\frac{\mu}{2}\left(t-t_{0}\right)\right\} .}
\end{aligned}
$$

Hence, the zero of the error system (3) is globally and exponentially stable. This completes the proof of Theorem 9 .

Remark 10. Recently, significant effort has been devoted to the study of the synchronization of complex dynamical networks [11-25]. However, the synchronization criteria in most of the existing studies contain unknown parameters, thereby making system synchronization difficult for workers to verify. In this study, a method for verifying system synchronization is obtained in Theorem 9 by constructing a simple Lyapunov function. The method relies only on the parameters of the system itself and does need any other adjustment parameter, thereby reducing delays and providing convenience for controller design.

\subsection{Global Exponential Synchronization of System (1) Using Generalized Halanay Inequalities}

Lemma 11 (see [35]). For any vector function $x(t), y(t) \in R^{n}$, $\bar{x}(t)=\sup _{t-\tau \leq s \leq t} x(s)$ and $\bar{y}(t)=\sup _{t-\tau \leq s \leq t} y(s)$ satisfy the following condition:

(1) $x(t)<y(t), t_{0}-\tau \leq t \leq t_{0}$.

(2) $D^{+} y(t)>F(t, y(t), \bar{y}(t)), t \geq t_{0} \geq 0 ; D^{+} x(t) \leq$ $F(t, x(t), \bar{x}(t)), t \geq t_{0} \geq 0$, where $F(t, x(t), \bar{x}(t))=$ $(A+B) x(t)+C \bar{x}(t), A<0$, is a diagonal matrix. Then, $x(t) \leq y(t), t \geq t_{0}$.
Theorem 12. If Assumption 7 holds and $M=-\left(p_{i i}+q_{i j}+\right.$ $\left.r_{i j}\right)_{N \times N}$ is an M-matrix, in which

$$
\begin{aligned}
& p_{i i}=-\varepsilon c_{1} l_{i}+\sigma_{i} ; \\
& q_{i j}= \begin{cases}c_{1} l_{j} c_{i j}, & i \neq j \\
0, & i=j ;\end{cases} \\
& r_{i j}=c_{2} l_{j}\left|d_{i j}\right|,
\end{aligned}
$$

$$
i, j=1,2, \ldots, N
$$

then the complex dynamical network (1) can achieve global exponential synchronization.

Proof. Taking the Dini derivative of $\left|e_{i}(t)\right|$, we obtain

$$
\begin{aligned}
D^{+}\left|e_{i}(t)\right|= & \dot{e}_{i} \operatorname{sgn} e_{i} \\
\leq & \left|f_{i}\left(x_{i}(t)\right)-f(s(t))\right| \\
& +c_{1} \sum_{j=1, j \neq i}^{N} c_{i j}\left|\phi_{j}\left(e_{j}(t)\right)\right|+c_{1} c_{i i}\left|\phi_{i}\left(e_{i}(t)\right)\right| \\
& +c_{2} \sum_{j=1}^{N}\left|d_{i j}\right|\left|\phi_{j}\left(e_{j}(t-\tau(t))\right)\right| \\
\leq & \left(-\varepsilon c_{1} l_{i}+\sigma_{i}\right)\left|e_{i}(t)\right|+\sum_{j=1, j \neq i}^{N} c_{1} l_{j} c_{i j}\left|e_{j}(t)\right| \\
& +\sum_{j=1}^{N} c_{2} l_{j}\left|d_{i j}\right|\left|\bar{e}_{j}(t)\right| \\
= & p_{i i}\left|e_{i}(t)\right|+\sum_{j=1}^{N} q_{i j}\left|e_{j}(t)\right|+\sum_{j=1}^{N} r_{i j}\left|\bar{e}_{j}(t)\right| \\
= & F(t, e(t), \bar{e}(t)), \quad i=1,2, \ldots, N .
\end{aligned}
$$

From the property of $M$-matrix, constants $\delta>0, \theta_{j}>0$, $(j=1,2, \ldots, N)$ exist such that

$$
p_{i i} \theta_{i}+\sum_{j=1}^{N}\left(q_{i j}+r_{i j}\right) \theta_{j}<-\delta, \quad i=1,2, \ldots, N .
$$

Let $0<\alpha \ll 1$, we can obtain

$$
\alpha \theta_{i}+p_{i i} \theta_{i}+\sum_{j=1}^{N}\left(q_{i j} \theta_{j}+r_{i j} \theta_{j} \exp (\alpha \tau)\right)<0 .
$$

When $t \in\left[t_{0}-\tau, t_{0}\right]$, let $R \gg 1$, such that

$$
R \theta_{i} \exp (-\alpha t)>1
$$

For any vector $\Upsilon=\left(\Upsilon_{1}, \Upsilon_{2}, \ldots, \Upsilon_{N}\right)^{T}>0$, we construct a vector function

$$
q_{i}(t)=R \theta_{i}\left[\sum_{j=1}^{N} \bar{e}_{j}\left(t_{0}\right)+\Upsilon\right] \exp \left\{-\alpha\left(t-t_{0}\right)\right\}
$$


Taking the Dini derivative of $\left|q_{i}(t)\right|$, we obtain

$$
\begin{aligned}
& D^{+}\left|q_{i}(t)\right| \\
& \quad=-\alpha R \theta_{i}\left[\sum_{j=1}^{N}\left|\bar{e}_{j}\left(t_{0}\right)\right|+\Upsilon\right] \exp \left\{-\alpha\left(t-t_{0}\right)\right\} .
\end{aligned}
$$

Substituting (19) into (22) yields

$$
\begin{aligned}
& D^{+}\left|q_{i}(t)\right|>\left[p_{i i} \theta_{i}+\sum_{j=1}^{N}\left(q_{i j} \theta_{j}+r_{i j} \theta_{j} \exp (\alpha \tau)\right)\right] \\
& \cdot R\left[\sum_{j=1}^{N}\left|\bar{e}_{j}\left(t_{0}\right)\right|+\Upsilon\right] \exp \left\{-\alpha\left(t-t_{0}\right)\right\} \\
& =p_{i i} \theta_{i} R\left[\sum_{j=1}^{N}\left|\bar{e}_{j}\left(t_{0}\right)\right|+\Upsilon\right] \exp \left\{-\alpha\left(t-t_{0}\right)\right\} \\
& +\sum_{j=1}^{N} q_{i j} \theta_{j} R\left[\sum_{j=1}^{N}\left|\bar{e}_{j}\left(t_{0}\right)\right|+\Upsilon\right] \exp \left\{-\alpha\left(t-t_{0}\right)\right\} \\
& \cdot \sum_{j=1}^{N} r_{i j} \theta_{j} R\left[\sum_{j=1}^{N}\left|\bar{e}_{j}\left(t_{0}\right)\right|+\Upsilon\right] \exp \left\{-\alpha\left(t-t_{0}\right)\right\} \\
& \cdot \exp (\alpha \tau) \geq p_{i i}\left|q_{i}(t)\right|+\sum_{j=1}^{N} q_{i j}\left|q_{j}(t)\right| \\
& +\sum_{j=1}^{N} r_{i j}\left|\bar{q}_{j}(t)\right|=F(t, q(t), \bar{q}(t)) .
\end{aligned}
$$

According to (20), when $t \in\left[t_{0}-\tau, t_{0}\right]$, we can derive

$$
\begin{aligned}
\left|q_{i}(t)\right| & =R \theta_{i}\left[\sum_{j=1}^{N}\left|\bar{e}_{j}\left(t_{0}\right)\right|+\Upsilon\right] \exp \left\{-\alpha\left(t-t_{0}\right)\right\} \\
& >\sum_{j=1}^{N}\left|\bar{e}_{j}\left(t_{0}\right)\right|+\Upsilon
\end{aligned}
$$

It is obvious that $\left|e_{i}(t)\right| \leq \sum_{j=1}^{N}\left|\bar{e}_{j}\left(t_{0}\right)\right|+\Upsilon, t \in\left[t_{0}-\tau, t_{0}\right]$; thus

$$
\left|e_{i}(t)\right|<\left|q_{i}(t)\right|, \quad t \in\left[t_{0}-\tau, t_{0}\right] .
$$

In view of (17), (23), and (25) and by using Lemma 11, we can derive

$$
\begin{aligned}
\left|e_{i}(t)\right| & <\left|q_{i}(t)\right| \\
& =R \theta_{i}\left[\sum_{j=1}^{N}\left|\bar{e}_{j}\left(t_{0}\right)\right|+\Upsilon\right] \exp \left\{-\alpha\left(t-t_{0}\right)\right\},
\end{aligned}
$$

Let $\Upsilon \rightarrow 0^{+}, \sum_{i=1}^{N} R \theta_{i}=\xi_{i}$, and we obtain

$$
\left|e_{i}(t)\right| \leq \xi_{i}\left[\sum_{j=1}^{N}\left|\bar{e}_{j}\left(t_{0}\right)\right|\right] \exp \left\{-\alpha\left(t-t_{0}\right)\right\}, \quad t \geq t_{0},
$$

where $\xi_{i}, \alpha$ are positive constants. According to Definition 3, the error system (3) is globally and exponentially stable, which implies that global exponential synchronization is achieved. This completes the proof of Theorem 12 .

Remark 13. Several synchronization criteria for the system are derived by constructing Lyapunov functions and using the Halanay inequality $[36,37]$. However, these synchronization criteria contain unknown parameters. In this paper, we avoid such issues by building the Lyapunov function. The Dini derivative loosen the requirements of the system state function; that is, it does not require the state function to be derivable. Moreover, certain points of the system are not derivable if the system is under discontinuous control. A direct solution of the error vector function for the Dini derivative can reduce the conservativeness of conditions. Moreover, a synchronization criterion is obtained in Theorem 12 by using generalized Halanay inequalities. The synchronization criterion relies only on the parameters of the system itself and does not contain any unknown adjustment parameter. Thus, our model provides convenience for practical applications.

Remark 14. In this paper, we provide conservative estimates of the size of $\alpha$ as it cannot be confirmed (Theorem 12); however, exact values are preferred.

Theorem 15. Supposing that the condition of Theorem 12 holds and constants $\varsigma>0$ exist such that $\widetilde{M}=-\left(\widetilde{p}_{i i}+q_{i j}+r_{i j}\right)_{N \times N}$ is still an M-matrix, one can derive

$$
\begin{array}{r}
\left|e_{i}(t)\right| \leq \widetilde{\xi}_{i}\left[\sum_{j=1}^{N}\left|\bar{e}_{j}\left(t_{0}\right)\right|\right] \exp \left\{-\varsigma\left(t-t_{0}\right)\right\}, \\
i=1,2, \ldots, N, t \geq t_{0},
\end{array}
$$

where

$$
\begin{aligned}
& \tilde{p}_{i i}=-\varepsilon c_{1} l_{i}+\sigma_{i}+\varsigma ; \\
& q_{i j}= \begin{cases}c_{1} l_{j} c_{i j}, & i \neq j \\
0, & i=j ;\end{cases} \\
& r_{i j}=c_{2} l_{j}\left|d_{i j}\right|,
\end{aligned}
$$


and then the complex dynamical network (1) can achieve global exponential synchronization with $\varsigma$ as the convergence rate.

Proof. Let $\left|y_{i}(t)\right|=\left|e_{i}(t)\right| \exp (\varsigma t), i=1,2, \ldots, N$. Taking the Dini derivative of $\left|y_{i}(t)\right|$, we obtain

$$
\begin{aligned}
& D^{+}\left|y_{i}(t)\right| \leq \varsigma \exp (\varsigma t)\left|e_{i}(t)\right|+\exp (\varsigma t) D^{+}\left|e_{i}(t)\right| \\
& \quad \leq \exp (\varsigma t)\left(\varsigma\left|e_{i}(t)\right|+p_{i i}\left|e_{i}(t)\right|+\sum_{j=1}^{N} q_{i j}\left|e_{j}(t)\right|\right. \\
& \left.+\sum_{j=1}^{N} r_{i j}\left|\bar{e}_{j}(t)\right|\right) \leq \widetilde{p}_{i i}\left|y_{i}(t)\right|+\sum_{j=1}^{N} q_{i j}\left|y_{j}(t)\right| \\
& +\sum_{j=1}^{N} r_{i j}\left|\bar{y}_{j}(t)\right| .
\end{aligned}
$$

Using Theorem 12, we obtain

$$
\left|y_{i}(t)\right| \leq \xi_{i}\left[\sum_{j=1}^{N}\left|\bar{y}_{j}\left(t_{0}\right)\right|\right] \exp \left\{-\alpha\left(t-t_{0}\right)\right\}, \quad t \geq t_{0} .
$$

Thus

$$
\begin{aligned}
\left|e_{i}(t)\right| & \leq \widetilde{\xi}_{i}\left[\sum_{j=1}^{N}\left|\bar{e}_{j}\left(t_{0}\right)\right|\right] \exp \left\{(-\alpha-\varsigma)\left(t-t_{0}\right)\right\} \\
& \leq \widetilde{\xi}_{i}\left[\sum_{j=1}^{N}\left|\bar{e}_{j}\left(t_{0}\right)\right|\right] \exp \left\{-\varsigma\left(t-t_{0}\right)\right\} .
\end{aligned}
$$

This completes the proof of Theorem 15.

If system (1) does not satisfy the conditions of the above theorem, that is, the system cannot achieve synchronization by itself, we can apply the appropriate controller to achieve synchronization.

Remark 16. On the basis of Theorem 12, we provide a simple and feasible method for calculating the exponential convergence rate in Theorem 15.

3.3. Global Exponential Synchronization of the Controlled System (1). The controlled complex dynamical network can be described as

$$
\begin{aligned}
\dot{x}_{i}(t)= & f\left(x_{i}(t)\right)+c_{1} \sum_{j=1}^{N} c_{i j} g_{j}\left(x_{j}(t)\right) \\
& +c_{2} \sum_{j=1}^{N} d_{i j} g_{j}\left(x_{j}(t-\tau(t))\right)+u_{i}(t),
\end{aligned}
$$

the controller $u_{i}(t)(i=1,2, \ldots, N)$ is designed as follows:

$$
u_{i}(t)=-k_{i} x_{i}(t)
$$

where $k_{i}>0$ is the adaptive feedback gain to be designed.

We obtain the error system on the basis of network (33) and (2) as follows:

$$
\begin{aligned}
\dot{e}_{i}(t)= & \dot{x}_{i}(t)-\dot{s}(t) \\
= & f\left(x_{i}(t)\right)-f(s(t))+c_{1} \sum_{j=1}^{N} c_{i j} \phi_{j}\left(e_{j}(t)\right) \\
& +c_{2} \sum_{j=1}^{N} d_{i j} \phi_{j}\left(e_{j}(t-\tau(t))\right)-k_{i} x_{i}(t),
\end{aligned}
$$

where $\phi_{i}\left(e_{i}(t)\right)=g_{i}\left(x_{i}(t)\right)-g_{i}(s(t)), \phi_{i}\left(e_{i}(t-\tau(t))\right)=g_{i}\left(x_{i}(t-\right.$ $\tau(t)))-g_{i}(s(t-\tau(t)))$.

Theorem 17. Supposing that Assumption 7 holds and satisfies the following condition:

$$
\begin{gathered}
\min _{1 \leq i \leq N} k_{i}+\min _{1 \leq i \leq N}\left(\varepsilon c_{1} l_{i}\right)>\max _{1 \leq i \leq N}\left(\sigma_{i}+\sum_{j=1, j \neq i}^{N} c_{1} l_{i} c_{i j}\right) \\
+2 \max _{1 \leq i \leq N} \frac{c_{2} \lambda l_{i}}{2},
\end{gathered}
$$

where $\lambda$ is the maximum eigenvalue of the matrix $D=$ $\left(d_{i j}\right)_{N \times N}$, then the complex dynamical network (33) can achieve global exponential synchronization. $\mu / 2$ is the convergence rate, where $\mu$ is the same as in Theorem 9.

A similar approach in Theorem 9 can be used for its proof.

Theorem 18. Supposing that Assumption 7 holds and $\bar{M}=$ $-\left(\bar{p}_{i i}+q_{i j}+r_{i j}\right)_{N \times N}$ is an M-matrix, where

$$
\begin{aligned}
& \bar{p}_{i i}=-k_{i}-\varepsilon c_{1} l_{i}+\sigma_{i} ; \\
& q_{i j}= \begin{cases}c_{1} l_{j} c_{i j}, & i \neq j \\
0, & i=j ;\end{cases} \\
& r_{i j}=c_{2} l_{j}\left|d_{i j}\right|,
\end{aligned}
$$

$$
i, j=1,2, \ldots, N
$$

then the complex dynamical network (33) can achieve global exponential synchronization.

A similar approach in Theorem 12 can be used for its proof. 
Theorem 19. Supposing that the condition of Theorem 18 holds and constants $\varsigma>0$ exist such that $\widetilde{\bar{M}}=-\left(\widetilde{\bar{p}}_{i i}+q_{i j}+r_{i j}\right)_{N \times N}$ is still an M-matrix, one can derive

$$
\begin{array}{r}
\left|e_{i}(t)\right| \leq \widetilde{\xi}_{i}\left[\sum_{j=1}^{N}\left|\bar{e}_{j}\left(t_{0}\right)\right|\right] \exp \left\{-\varsigma\left(t-t_{0}\right)\right\}, \\
i=1,2, \ldots, N, t \geq t_{0},
\end{array}
$$

where

$$
\begin{aligned}
& \bar{p}_{i i}=-k_{i}-\varepsilon c_{1} l_{i}+\sigma_{i}+\varsigma ; \\
& q_{i j}= \begin{cases}c_{1} l_{j} c_{i j}, & i \neq j \\
0, & i=j ;\end{cases} \\
& r_{i j}=c_{2} l_{j}\left|d_{i j}\right|,
\end{aligned}
$$

$$
i, j=1,2, \ldots, N \text {. }
$$

The complex dynamical network (33) can then achieve global exponential synchronization with $\varsigma$ as the convergence rate. proof.

A similar approach in Theorem 15 can be used for its

Remark 20. We provide several synchronization criteria for adding the controller in Theorems 17, 18, and 19. The feedback gain $k_{i}$ of the controllers is related only to the inherent parameters of the system.

\section{Numerical Simulation}

A dynamical network model with 10 dynamical nodes is considered. The state of the $i$ th node is as presented in (1). Without loss of generality, we choose the system parameters that satisfy Theorem 15, that is, $c_{1}=c_{2}=1, \sigma=2, l=2$ after considering the coupling matrices:

C

$$
=\left(\begin{array}{cccccccccc}
-4 & 1 & 0 & 0 & 1 & 0 & 0 & 0 & 1 & 1 \\
1 & -4 & 1 & 1 & 1 & 0 & 0 & 0 & 0 & 0 \\
0 & 1 & -4 & 1 & 0 & 1 & 0 & 0 & 1 & 0 \\
0 & 1 & 1 & -4 & 0 & 0 & 1 & 1 & 0 & 0 \\
1 & 1 & 0 & 0 & -4 & 1 & 0 & 0 & 0 & 1 \\
0 & 0 & 1 & 0 & 1 & -4 & 0 & 1 & 0 & 1 \\
0 & 0 & 0 & 1 & 0 & 0 & -4 & 1 & 1 & 1 \\
0 & 0 & 0 & 1 & 0 & 1 & 1 & -4 & 1 & 0 \\
1 & 0 & 1 & 0 & 0 & 0 & 1 & 1 & -4 & 0 \\
1 & 0 & 0 & 0 & 1 & 1 & 1 & 0 & 0 & -4
\end{array}\right),
$$

$D$

$$
=\left(\begin{array}{cccccccccc}
-3 & 1 & 0 & 0 & 0 & 0 & 0 & 1 & 1 & 0 \\
0 & -2 & 1 & 0 & 0 & 0 & 1 & 0 & 0 & 0 \\
1 & 0 & -1 & 0 & 0 & 0 & 0 & 0 & 0 & 0 \\
0 & 0 & 0 & -1 & 1 & 0 & 0 & 0 & 0 & 1 \\
0 & 0 & 0 & 1 & -3 & 0 & 1 & 1 & 0 & 0 \\
0 & 0 & 0 & 0 & 0 & -1 & 0 & 0 & 0 & 1 \\
0 & 0 & 0 & 0 & 1 & 1 & -3 & 0 & 0 & 0 \\
1 & 1 & 0 & 0 & 1 & 0 & 0 & -3 & 0 & 0 \\
0 & 0 & 0 & 0 & 0 & 0 & 0 & 0 & -1 & 1 \\
1 & 0 & 0 & 0 & 0 & 0 & 1 & 0 & 0 & -2
\end{array}\right) .
$$

Figure 1 illustrates the trajectories of a system error. The error states are exponentially stable at zero equilibrium points, thereby implying that global exponential synchronization was obtained by the dynamic nodes of the complex dynamical network with time-varying delays.

The parameters of the system are adjusted such that the conditions of Theorem 15 are not established. Figures 2, 4, and 6 show the trajectories of an error system with unstable error states. According to Theorem 19, we added the feedback controller (34) to the time-varying delay complex network (33). Figures 3, 5, and 7 show the obtained trajectories of the error system. When the control gain $k_{i}>5$, the complex dynamical network can achieve global exponential synchronization.

\section{Conclusion}

In this study, several simple and generic synchronization criteria are derived to guarantee that a complex dynamical network achieves global exponential synchronization. These criteria do not contain unknown parameters related to the intrinsic parameters of the system. The specific form of time delay is unknown, thereby making practical application convenient. If the system cannot establish synchronization by itself, adding appropriate feedback controllers would ensure that sufficient conditions for system synchronization are obtained. The gain of the controllers is related only to the intrinsic parameters of the system itself. The estimation of exponential convergence rate has also been studied. Hence, a method for estimating the exponential convergence rate is also presented in this paper. For instance, solving transcendental equation (9) to obtain the exponential convergence rate is easier using related mathematical software. Theorems 15 and 19 also illustrate a simple and feasible method for calculating the exponential convergence rate. 


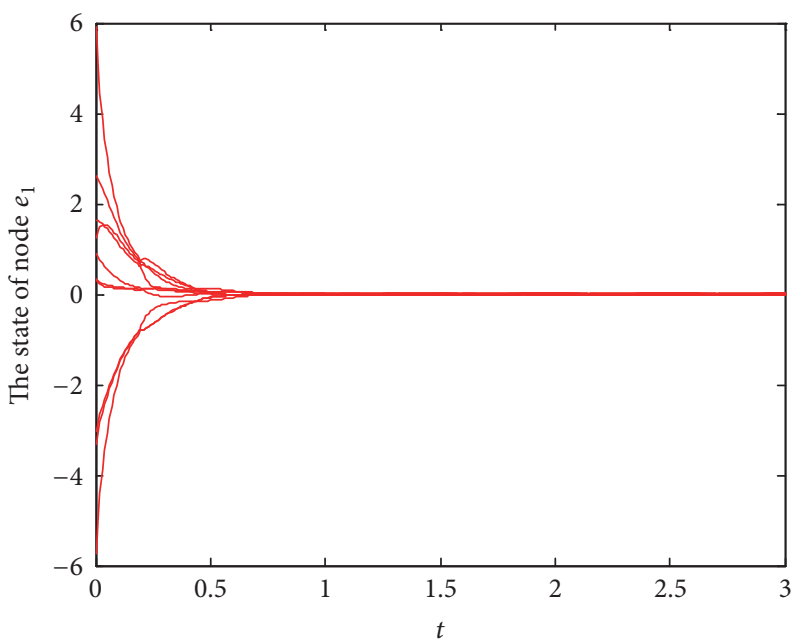

(a)

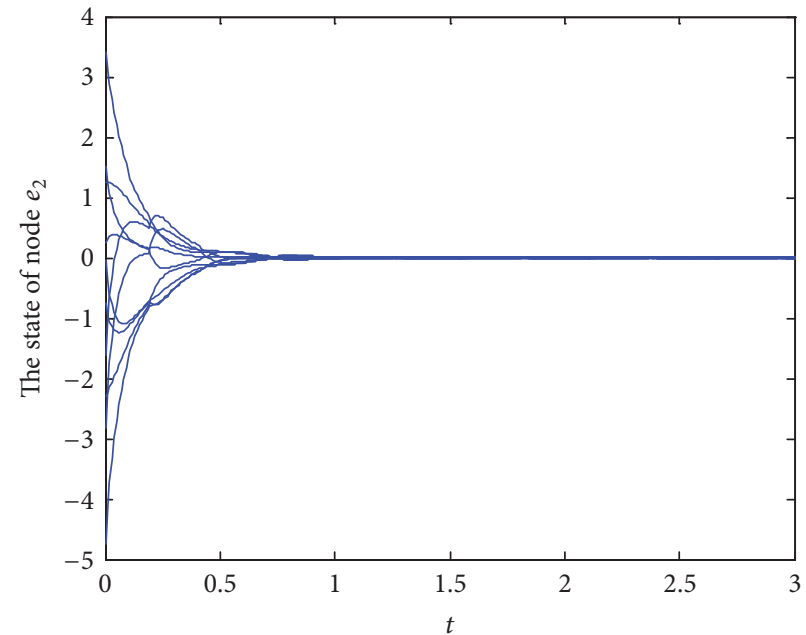

(b)

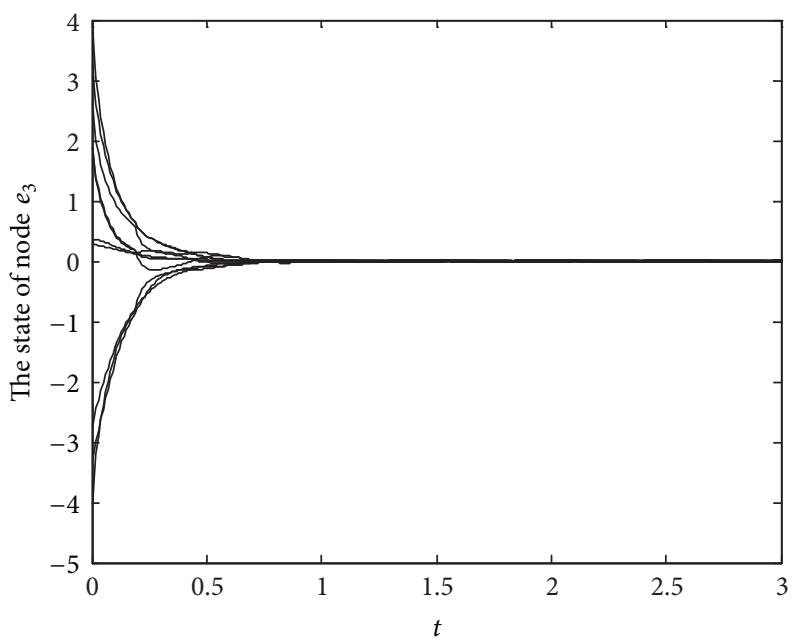

(c)

FIGURE 1: Synchronization error system that satisfies the conditions.

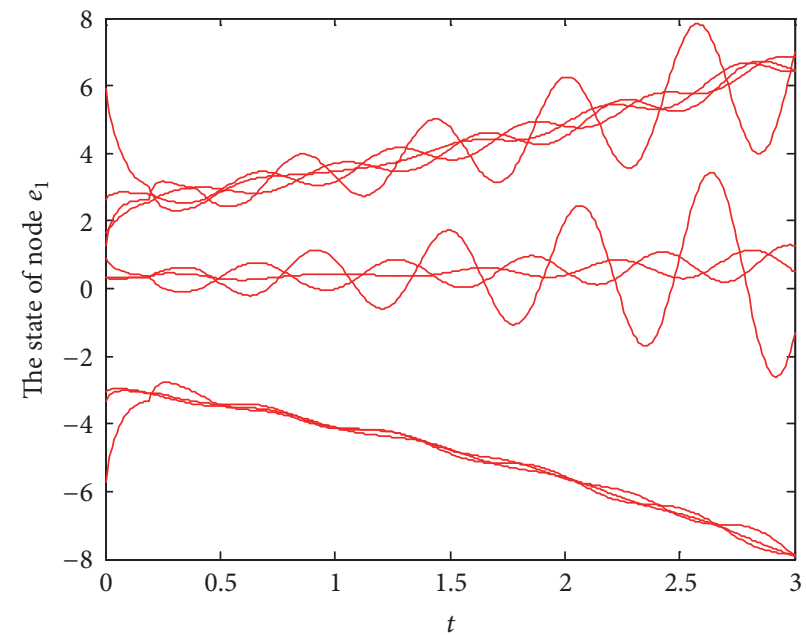

FIGURE 2: Synchronization error $e_{i 1}$ that does not satisfy the conditions. 


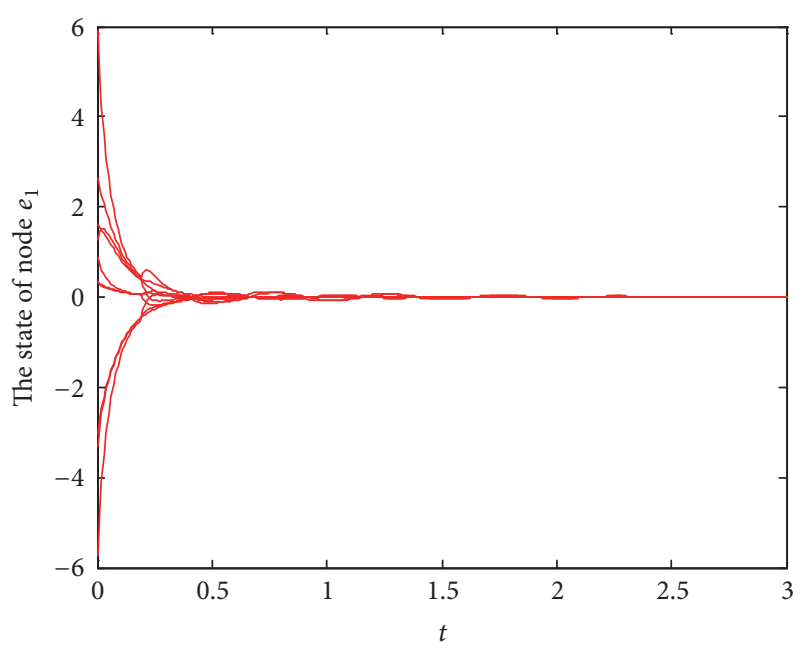

FIGURE 3: Synchronization error $e_{i 1}$ of adding controllers.

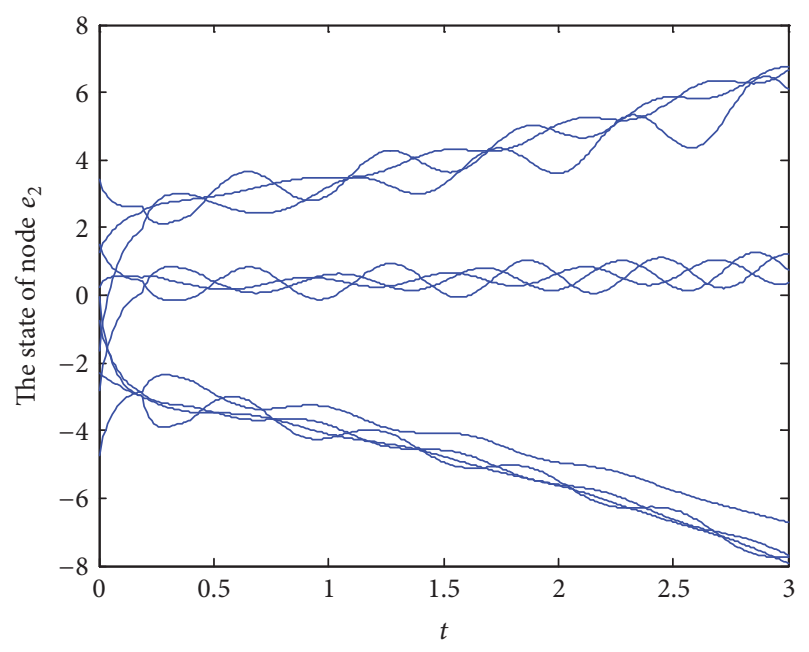

FIgURE 4: Synchronization error $e_{i 2}$ that does not satisfy the conditions.

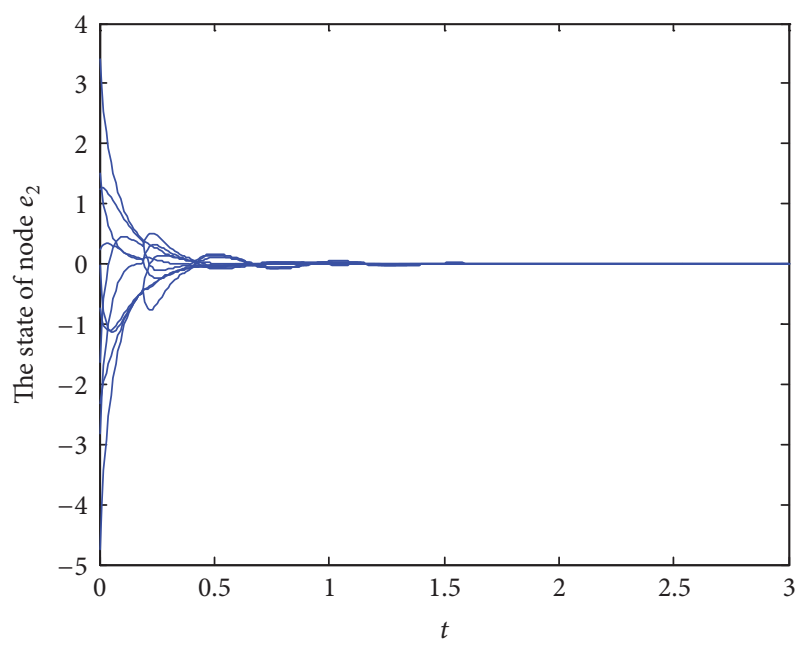

FIGURE 5: Synchronization error $e_{i 1}$ of adding controllers.

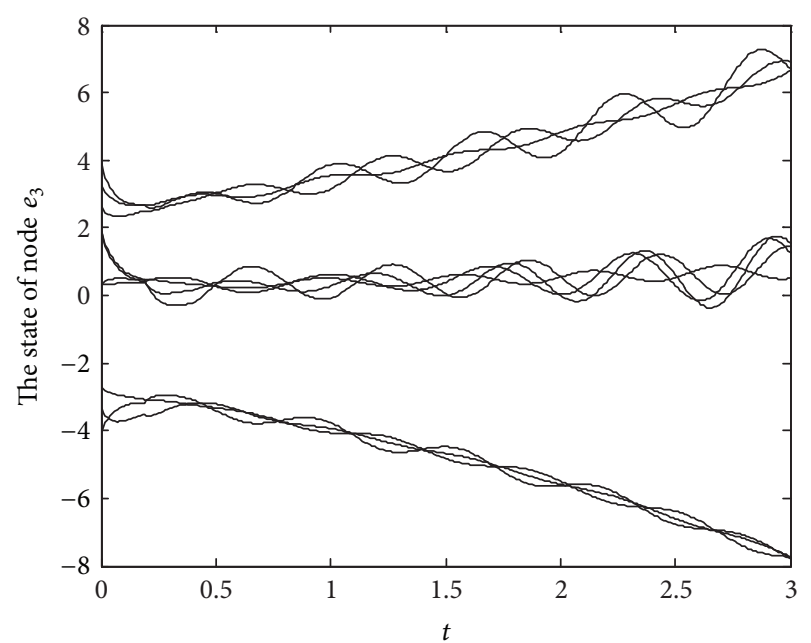

FIgURE 6: Synchronization error $e_{i 3}$ that does not satisfy the conditions.

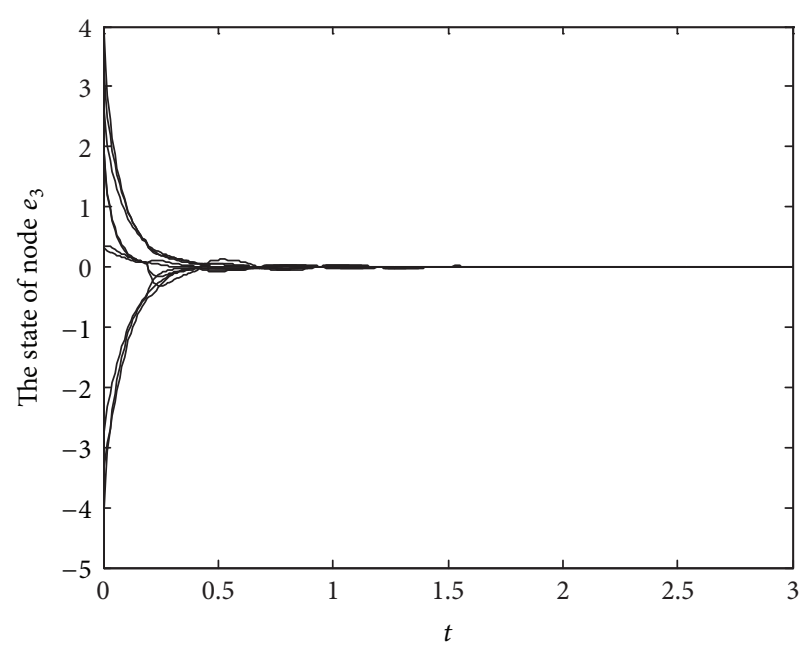

FIGURE 7: Synchronization error $e_{i 3}$ of adding controllers.

\section{Conflicts of Interest}

The authors (Yi-Ping Luo, Li Shu, and Bi-Feng Zhou) declare that there are no conflicts of interest regarding the publication of this paper.

\section{Acknowledgments}

This work was jointly supported by the National Natural Science Foundation of China (Grant no. 11372107) and Natural Science Foundation of Hunan Province (Grant no. 2017JJ4004).

\section{References}

[1] A. L. Barabási, R. Albert, and H. Jeong, "Mean-field theory for scale-free random networks," Physica A: Statistical Mechanics and its Applications, vol. 272, no. 1, pp. 173-187, 1999.

[2] S. Boccaletti, V. Latora, Y. Moreno, M. Chavez, and D. W. Hwang, "Complex networks: structure and dynamics," Physics Reports, vol. 424, no. 4-5, pp. 175-308, 2006. 
[3] L. D. F. Costa, O. N. Oliveira, G. Travieso et al., "Analyzing and modeling real-world phenomena with complex networks: a survey of applications," Advances in Physics, vol. 60, no. 3, pp. 329-412, 2011.

[4] X. F. Wang and G. Chen, "Complex networks: small-world, scale-free and beyond," IEEE Circuits and Systems Magazine, vol. 3, no. 1, pp. 6-20, 2003.

[5] A. Arenas, A. D. Guilera, J. Kurths, Y. Moreno, and C. Zhou, "Synchronization in complex networks," Physics Reports, vol. 469, no. 3, pp. 93-153, 2008.

[6] A. Khan and M. A. Bhat, "Multi-switching combination-combination synchronization of non-identical fractional-order chaotic systems," Mathematical Methods in the Applied Sciences, 2017.

[7] S. Zheng, "Synchronization analysis of time delay complexvariable chaotic systems with discontinuous coupling," Journal of the Franklin Institute. Engineering and Applied Mathematics, vol. 353, no. 6, pp. 1460-1477, 2016.

[8] L. M. Pecora and T. L. Carroll, "Master stability functions for synchronized coupled systems," Physical Review Letters, vol. 80, no. 10, pp. 2109-2112, 1998.

[9] H.-L. Li, Z. Wang, Y.-L. Jiang, L. Zhang, and Z. Teng, "Antisynchronization and intermittent anti-synchronization of two identical delay hyperchaotic chua systems via linear control," Asian Journal of Control, vol. 19, no. 1, pp. 202-214, 2017.

[10] S. Cai, P. Zhou, and Z. Liu, "Synchronization analysis of hybridcoupled delayed dynamical networks with impulsive effects: a unified synchronization criterion," Journal of the Franklin Institute, vol. 352, no. 5, pp. 2065-2089, 2015.

[11] T. Hu, "Hybrid synchronization and parameter identification of uncertain interacted networks," Optik, vol. 127, no. 19, pp. 75577564, 2016.

[12] B. Li, "Hybrid synchronization of two complex delayed dynamical networks with nonidentical topologies and mixed coupling," Complexity, vol. 21, no. S2, pp. 470-482, 2016.

[13] J. Lu, D. W. C. Ho, and J. Cao, "A unified synchronization criterion for impulsive dynamical networks," Automatica, vol. 46, no. 7, pp. 1215-1221, 2010.

[14] X. F. Wang and G. Chen, "Synchronization in small-world dynamical networks," International Journal of Bifurcation and Chaos in Applied Sciences and Engineering, vol. 12, no. 1, pp. 187192, 2002.

[15] X. F. Wang and G. Chen, "Synchronization in scale-free dynamical networks: robustness and fragility," IEEE Transactions on Circuits and Systems I: Fundamental Theory and Applications, vol. 49, no. 1, pp. 54-62, 2002.

[16] Y. Liu, Z. Wang, J. Liang, and X. Liu, "Synchronization and state estimation for discrete-time complex networks with distributed delays," IEEE Transactions on Systems, Man, and Cybernetics B, vol. 38, no. 5, pp. 1314-1325, 2008.

[17] C. Huang, D. W. C. Ho, and J. Lu, "Partial-information-based synchronization analysis for complex dynamical networks," Journal of the Franklin Institute, vol. 352, no. 9, pp. 3458-3475, 2015.

[18] T. H. Lee, J. H. Park, D. H. Ji, O. M. Kwon, and S. Lee, “Guaranteed cost synchronization of a complex dynamical network via dynamic feedback control," Applied Mathematics and Computation, vol. 218, no. 11, pp. 6469-6481, 2012.

[19] J. Zhou, J. Lu, and J. Lv, "Adaptive synchronization of an uncertain complex dynamical network," IEEE Transactions on Automatic Control, vol. 51, no. 4, pp. 652-656, 2006.
[20] L. Zhang, X. Yang, C. Xu, and J. Feng, "Exponential synchronization of complex-valued complex networks with timevarying delays and stochastic perturbations via time-delayed impulsive control," Applied Mathematics and Computation, vol. 306, pp. 22-30, 2017.

[21] R. Cheng, M. Peng, and W. Yu, "Pinning synchronization of delayed complex dynamical networks with nonlinear coupling," Physica A: Statistical Mechanics and its Applications, vol. 413, pp. 426-431, 2014.

[22] X. Liu and T. Chen, "Cluster synchronization in directed networks via intermittent pinning control," IEEE Transactions on Neural Networks, vol. 22, no. 7, pp. 1009-1020, 2011.

[23] H. Dai, W. Chen, J. Jia, J. Liu, and Z. Zhang, "Exponential synchronization of complex dynamical networks with timevarying inner coupling via event-triggered communication," Neurocomputing, vol. 245, pp. 124-132, 2017.

[24] J.-a. Wang, X. Ma, X. Wen, and Q. Sun, "Pinning lag synchronization of drive-response complex networks via intermittent control with two different switched periods," Physica A. Statistical Mechanics and its Applications, vol. 461, pp. 278-287, 2016.

[25] S. Cai, X. Lei, and Z. Liu, "Outer synchronization between two hybrid-coupled delayed dynamical networks via aperiodically adaptive intermittent pinning control," Complexity, vol. 21, pp. 593-605, 2016.

[26] T. Liu, G. M. Dimirovski, and J. Zhao, "Exponential synchronization of complex delayed dynamical networks with general topology," Physica A: Statistical Mechanics and its Applications, vol. 387, no. 2-3, pp. 643-652, 2008.

[27] X. Wu and H. Lu, "Exponential synchronization of weighted general delay coupled and non-delay coupled dynamical networks," Computers \& Mathematics with Applications, vol. 60, no. 8, pp. 2476-2487, 2010.

[28] X. X. Liao and D. M. Xiao, "Globally exponential stability of hopfield neural networks with time-varying delays," Acta Electronica Sinica, vol. 28, pp. 87-90, 2000.

[29] J. Lü and G. Chen, "A time-varying complex dynamical network model and its controlled synchronization criteria," IEEE Transactions on Automatic Control, vol. 50, no. 6, pp. 841-846, 2005.

[30] M. Fang, "Synchronization for complex dynamical networks with time delay and discrete-time information," Applied Mathematics and Computation, vol. 258, pp. 1-11, 2015.

[31] P. He, X.-L. Wang, and Y. Li, "Guaranteed cost synchronization of complex networks with uncertainties and time-varying delays," Complexity, vol. 21, no. 6, pp. 381-395, 2016.

[32] A. Selivanov, A. Fradkov, and E. Fridman, "Passification-based decentralized adaptive synchronization of dynamical networks with time-varying delays," Journal of the Franklin Institute, vol. 352, no. 1, pp. 52-72, 2015.

[33] X. X. Liao, Theory and Application of Stability for Dynarnical Systems, National Defense Industry Preae, Beijing, China, 2000.

[34] A. Halanay, Differential Equations: Stability, Oscillations, Time Lags, Academic Press, New York, NY, USA, 1966.

[35] Y. P. Luo, F. Q. Deng, and A. P. Li, “Gobal expenential stabilization for parabolic type systems with continuously distributed delays," Acta Physica Sinica, vol. 56, pp. 637-642, 2007.

[36] S. Cai, Q. He, J. Hao, and Z. Liu, "Exponential synchronization of complex networks with nonidentical time-delayed dynamical nodes," Physics Letters. A, vol. 374, no. 25, pp. 2539-2550, 2010.

[37] M. Zhao, H. Zhang, Z. Wang, and H. Liang, "Synchronization between two general complex networks with time-delay by adaptive periodically intermittent pinning control," Neurocomputing, vol. 144, pp. 215-221, 2014. 


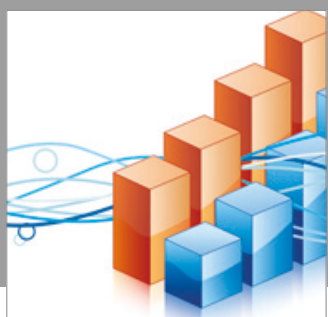

Advances in

Operations Research

vatersals

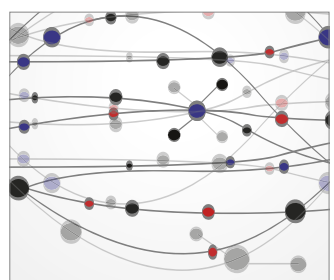

\section{The Scientific} World Journal
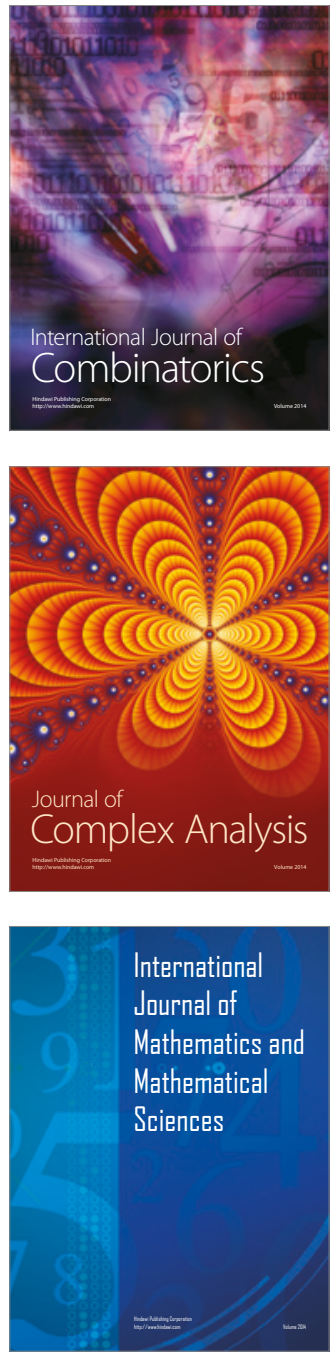
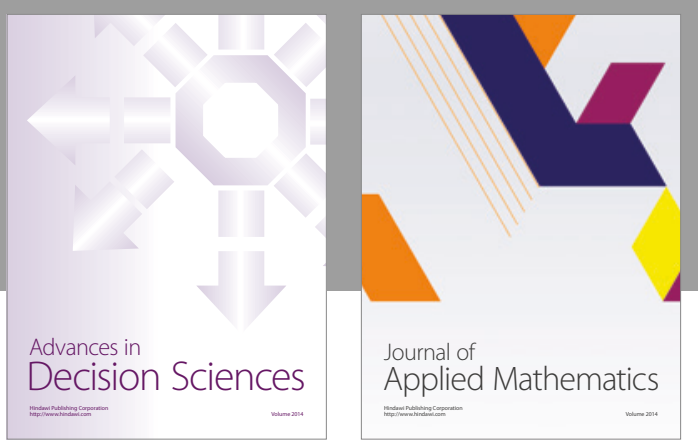

Algebra

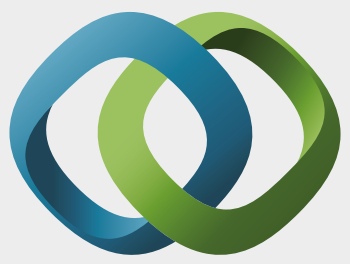

\section{Hindawi}

Submit your manuscripts at

https://www.hindawi.com
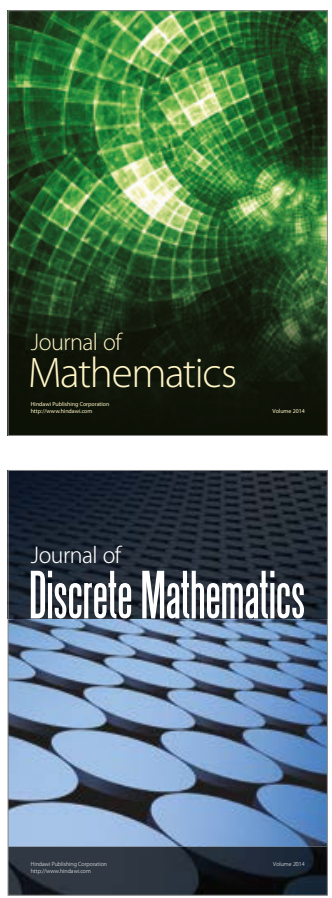

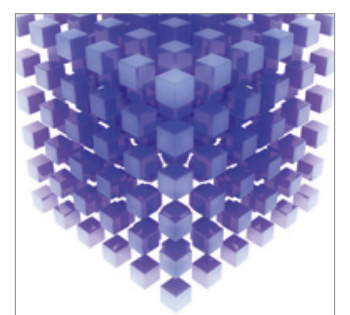

Mathematical Problems in Engineering
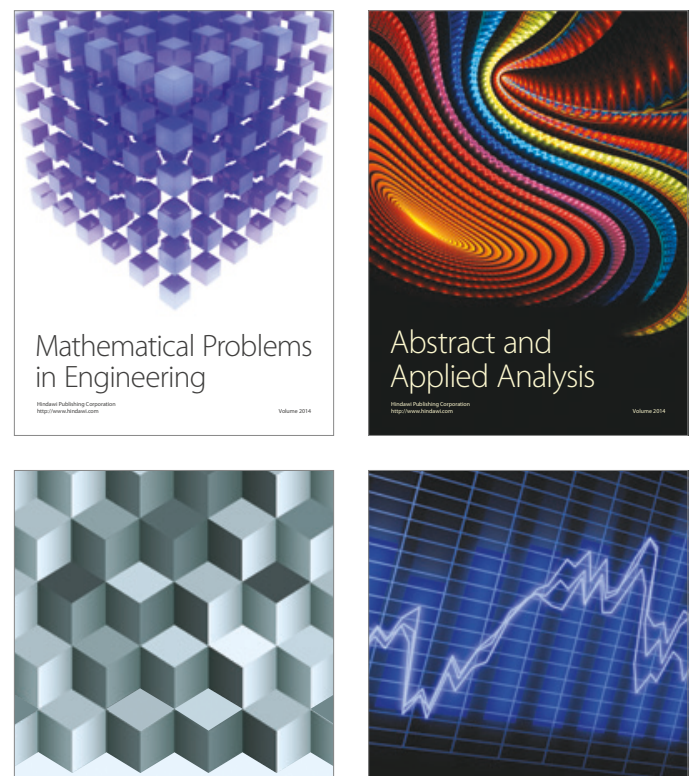

Journal of

Function Spaces

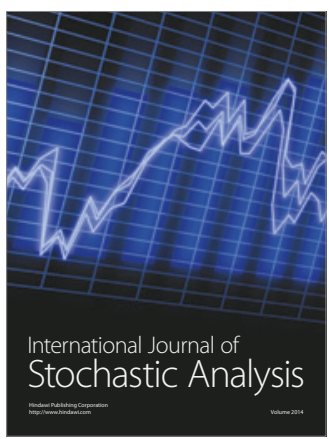

Probability and Statistics
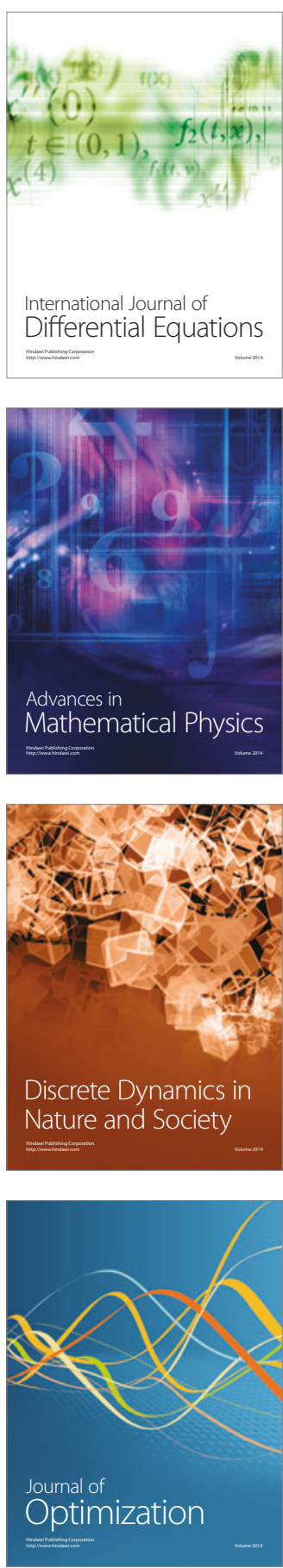